\title{
Gender Decision through Brain Economics
}

\author{
Mrs. Mary Cecil, and Prof.Samir Kumar Bandyopadhyay \\ 1Research Scholar of NHSM Knowledge Campus, Kolkata, India \\ 2Senior Member IEEE Dept. of Computer Science \& Engineering,University of Calcutta, India
}

\begin{abstract}
The emergence of behavioural economics has certainly led to much greater realism in the description of human gender decision making. There is the common questions which arise commonly in minds. The human brain is a highly complex organ. Studies of perception, cognition, memory and neural functions have found apparent gender differences. In this paper we review how gender difference arose from brain structure.
\end{abstract}

Key Words: - WM, GM, Neuroscience and Grey matter

\section{INTRODUCTION}

Behavioural economics has mostly been informed by a branch of psychology called "behavioural decision research," but other cognitive sciences are ripe for harvest. Some important insights will surely come from neuroscience, either directly or because neuroscience will reshape what is believed about psychology which in turn informs economics. Neuroscience uses imaging of brain activity and other techniques to infer details about how the brain works. The brain is the ultimate "black box." The foundations of economic theory were constructed assuming that details about the functioning of the brain's black box would not be known [1-4].

Although economists may privately acknowledge that actual flesh-and-blood human beings often choose without much deliberation, the economic models as written invariably represent decisions in a "deliberative equilibrium," i.e., that are at a stage where further deliberation, computation, reflection, etc. would not by itself alter the agent's choice. The variables that enter into the the decision problem - the preferences, information, and constraints - are precisely the variables that should affect the decision, if the person had unlimited time and computing ability[5-7].

The adult human brain weighs on average about $3 \mathrm{lb}(1.5 \mathrm{~kg})$ [1] with a size of around $1130 \mathrm{~cm} 3 \mathrm{in}$ women and $1260 \mathrm{~cm} 3$ in men although there is substantial individual variation [2]. Male brains are about 10\% larger than female brains and weigh 11-12\% more than that of a woman [8-9]. Men's heads are also about $2 \%$ bigger than women's. This is due to the larger physical stature of men. Male's larger muscle mass and larger body size requires more neuron to control them. The brain weight is related to the body weight partly because it increases with increasing height [3]. This difference is also present at birth. A boy's brain is between 12-20\% larger than that of a girl. The head circumference of boys is also larger (2\%) than that of girls. However, when the size of the brain is compared to body weight at this age, there is almost no difference between boys and girls. So, a girl baby and a boy baby who weigh the same will have similar brain sizes.

\section{GREY MATTER AND WHITE MATTER}

Ratios of grey to white matter differ significantly between the sexes in various regions of the human cortex [5]. Variations in the amount of white and grey matter in the brain remain significant [6-8]. Men have approximately 6.5 times more grey matter in the brain than women, and women have about 10 times more white matter than men do [3]. At the age of 20, a man has around 176,000 km and a woman of 149,000 km of myelinated axons in their brains [9].

Men appear to have more grey matter, made up of active neurons, and women more of the white matter responsible for communication between different areas of the brain [10].In women's brains, the neurons are packed in tightly, so that they're closer together. Some women even have as many as $12 \%$ more neurons than men do [10]. These neurons are densely crowded on certain layers of the cortex, namely the ones responsible for signals coming in and out of the brain, and these differences were present from birth [10]. When controlling for total cerebral volume, women had a higher percentage of grey matter than men, and men had a higher percentage of white matter $[6,8]$ and both grey and white matter volumes correlated with cognitive performance across sex groups. The average number of neocortical neurons was 19 billion in female brains and 23 billion in male brains, a $16 \%$ difference. In a study, which covered the age range from 20 years to 90 years, approximately $10 \%$ of all neocortical neurons are lost over the life span in both sexes. Sex and age were the main determinants of the total number of neurons in the human neocortex, whereas body size, per se, had no influence on neuron number [11]. Gender differences in precentral, cingulate, and anterior temporal white matter areas were also found, suggesting 
that microstructural white matter organization in these regions may have a sexual dimorphism [12]. So WM and GM may one the criterion for determining gender. We now give the procedure for finding WM and GM for a person.

\section{NOISE REDUCTION}

Before going further processing noise reduction is necessary. The concept of median is used for noise reduction. In case of mean or average filter replace the center of the pixel by mean (average) value of the pixels. Thus if a square window of size $2 \mathrm{k}+1$ is used, where $\mathrm{k}$ within 1 to $\mathrm{n}$, odd width and height are taking and average value is replaced by $(\mathrm{k}+1, \mathrm{k}+1)$ position. The basic function for median is written below in equation $(2)$, where $f(x, y)$ output mean and $g(x, y)$ is the original values.

$$
f(x, y)=\frac{1}{K} \sum_{i=1}^{K} g_{i}(x, y)
$$

Each time of scan value of central pixel of window is replaced by the average value of its neighbouring pixels comes within the window. If $\mathrm{k}=1$ the window size is $3 \times 3$ and center position is $2 \times 2$ is shown in figure 5 Before filtering if the intensity value are $46,58,47,49,41,45,42,55,58$. So the average value is 49 and then 41 are replaced by 49 .

\begin{tabular}{|l|l|l|}
\hline 46 & 58 & 47 \\
\hline 49 & 41 & 45 \\
\hline 42 & 55 & 58 \\
\hline \multicolumn{3}{|c|}{ (a) }
\end{tabular}

\begin{tabular}{|l|l|l|}
\hline 46 & 58 & 47 \\
\hline 49 & 49 & 45 \\
\hline 42 & 55 & 58 \\
\hline \multicolumn{3}{|c|}{ (b) }
\end{tabular}

Figure 1 : Mean filter: a) Shows pixel intensity value before filtering and b) Shows pixel intensity value after filtering.

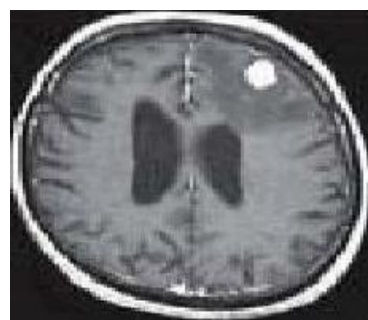

(a)

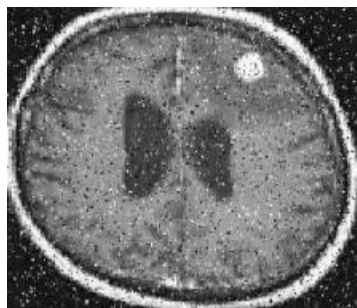

(e)

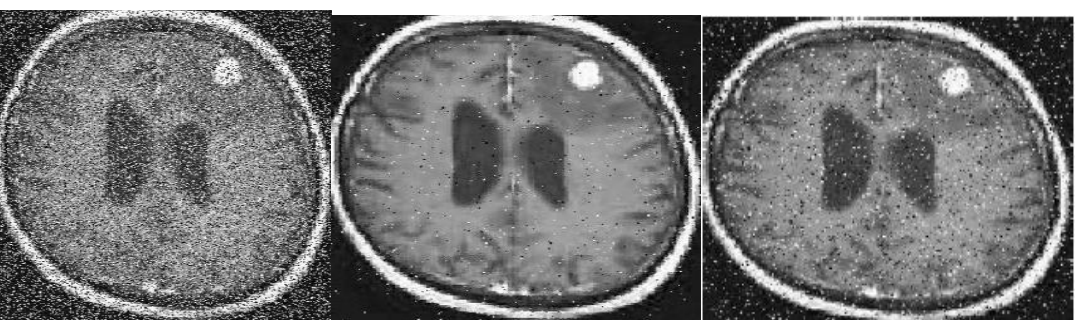

(c)

(d)

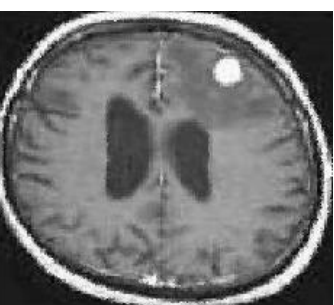

(f)

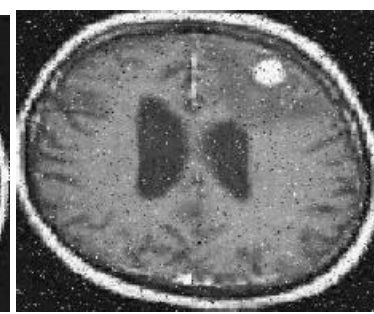

(g)

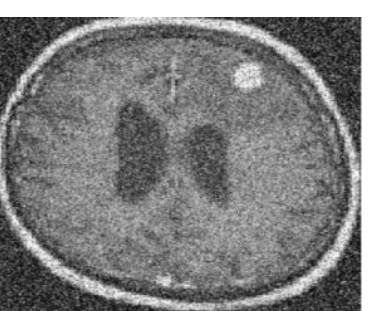

(h)

Figure 2 : A set of MRI of brain with different filtering output for the same image Output cited by Manohar Annappa Koli a) original MRI image, b) original MRI with 50\% noise, c) median filter, d) Min-Max Median Filter, e) Center Weighted Median Filter, f) Adaptive Median Filter, g) Progressive Switching Median Filter, h) Average Filter.

\section{Procedure for White matter and Gray matter Extraction}

The following procedure is used to find out GM and WM for gender calculation

Begin

Step 1: Input a gray scale image $I(x, y) ; x$ and $y$ being spatial coordinates of the image.

Step 2: B = STATBIN(Image I);

/* compute the binarized image*/

Step 3: FOR $\mathrm{i}=0$ to $\mathrm{x}$ DO

$$
\text { FOR } \mathrm{i}=0 \text { to } \mathrm{y} \text { DO }
$$




\section{IF $B(i, j)=1$ THEN \\ ELSE set $\mathrm{B}(\mathrm{i}, \mathrm{j}) \leftarrow 0$ \\ END IF \\ set $\mathrm{B}(\mathrm{i}, \mathrm{j}) \leftarrow 1$ \\ END FOR \\ END FOR \\ I* Complement of the image has been done */}

Step4: Compute two dimensional wavelet decomposition is done using ' $\mathrm{db1}$ ' wavelet up to level two.

I* this step has been done using [c1,s1] = wavedec2(B,2,'db1') matlab function */ Step5: Re-composition of the image is done using the approximate coefficient of previous step.

I* this step has been done using $\mathrm{RC}=$ appcoef2 $\left(\mathrm{c} 1, \mathrm{~s} 1, \mathrm{db}^{\prime}, 2\right) * /$

Step6: FOR $i=0$ to $x$ DO

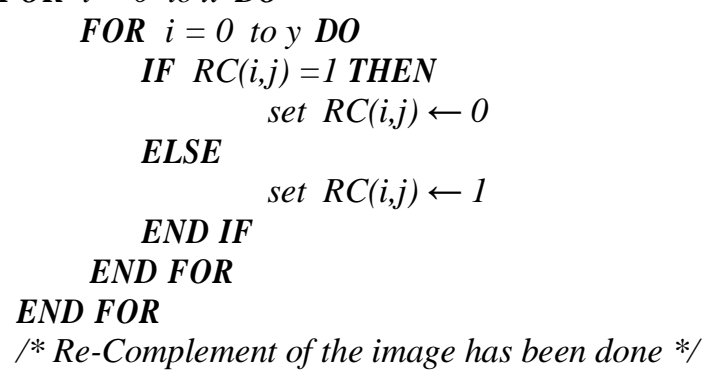

Step7: Labeling of the image RC is done using union find method and calculates area for each connected component and store in to ALLAREA.

Step8: $\mathrm{Z}=\mathbf{M A X}($ ALLAREA);

/* the maximum area of all the connected components is found out which represents the brain and the image obtained contains only the brain as 1 pixel */

Step9: A quick hull algorithms for convex hull is applied.

$/ *$ Convex hull is computed for these 1 pixel and the entire pixels inside hull set to 1

*/

Step10: GRAYIMAGE1 = I* BConvex

/* Convexed image is multiplied by original image */

Step11: BIN2=STATBIN(GRAYIMAGE1);

Step12: GRAYIMAGE2 = BIN2*GRAYIMAGE1

/* pixel wise multiplication has been done */

Step13: set sum $\leftarrow 0$ and set count $\leftarrow 0$

Step14: FOR $\mathrm{i}=0$ to $\mathrm{x}$

DO

FOR $\mathrm{j}=0$ to $\mathrm{y}$

DO

IF GRAYIMAGE2(i,j) $>0$ THEN

intensity $\leftarrow$ GRAYIMAGE2(i,j)

sum $\leftarrow$ sum + intensity

ENDIF

count $\leftarrow$ count +1

ENDFOR

\section{ENDFOR}

Step15: average $\leftarrow$ sum/count

Step16: white $\leftarrow$ zeros(a111,b111)

/* set all pixel of the white matrix zero */

Step17: grayŁzeros(a111,b111)

$1 *$ set all pixel of the gray matrix zero *

Step18: $\quad$ FOR $\mathrm{i}=0$ to $\mathrm{x}$

DO

$$
\begin{array}{ll}
\text { FOR } \mathrm{j}=\text { to } \mathrm{y} & \multicolumn{1}{c}{\text { DO }} \\
\text { IF } \mathrm{i}(\mathrm{i}, \mathrm{j})>0 & \text { THEN } \\
& \text { IF GRAYIMAGE2 }(\mathrm{i}, \mathrm{j})>\text { average } \\
& \text { white }(\mathrm{i}, \mathrm{j}) \leftarrow 1
\end{array}
$$




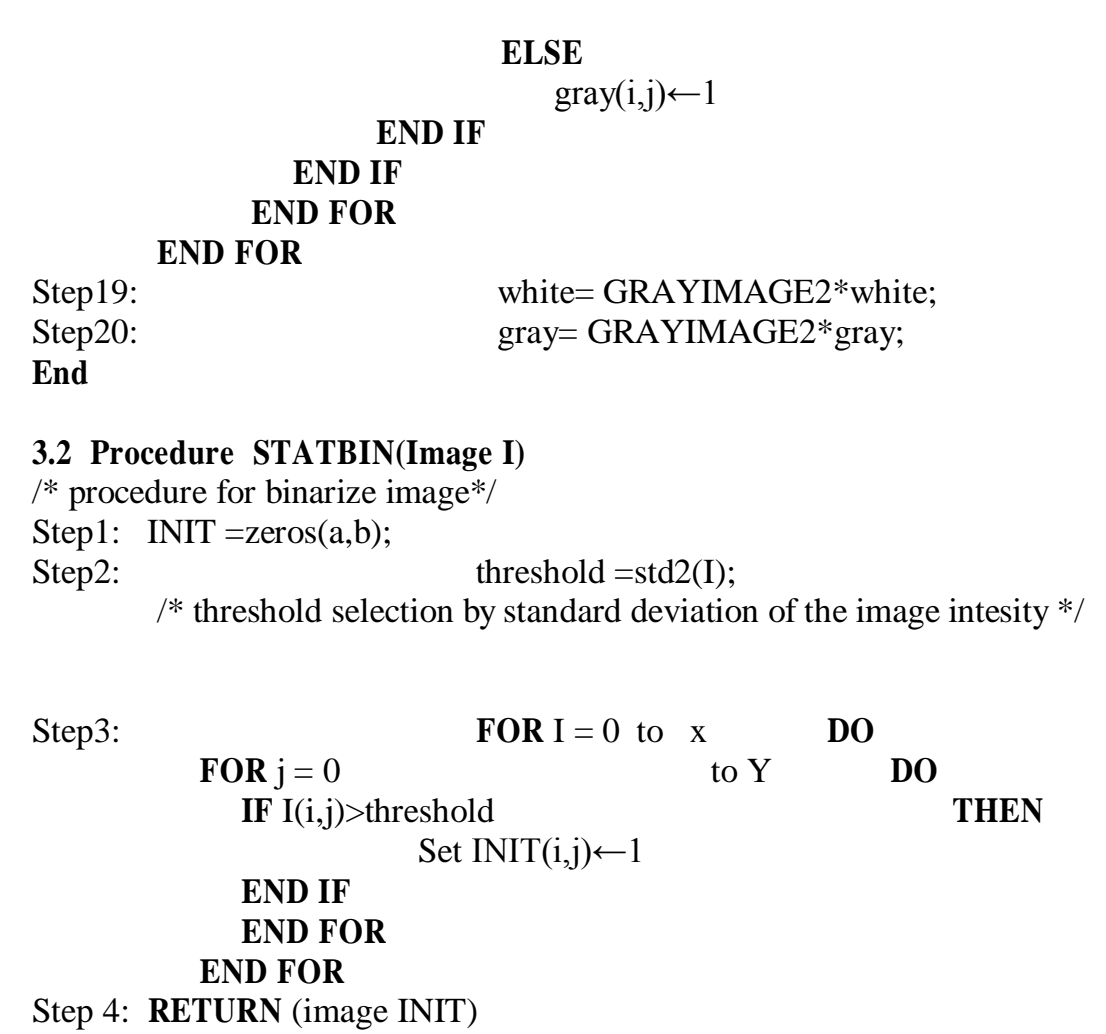

\section{CONCLUSIONS}

It is true that only GM and WM may not be the actual factor for finding gender but it indicates gender and our next task to include more factors for accurate finding of gender.

[1] Parent A, Carpenter MB. Carpenter's human neuroanatomy. Baltimore: Williams \& Wilkins 1995.

[2] Cosgrove KP, Mazure CM, Staley JK. Evolving knowledge of sex differences in brain structure, function, and chemistry. Biol Psychiatry 2007; 62: 847-55.

[3] Ho KC, Roessmann U, Straumfjord JV, Monroe G. Analysis of brain weight. II. Adult brain weight in relation to body height, weight, and surface area. Arch Pathol Lab Med 1980; 104(12): 640-5.

[4] Goldstein JM, Seidman LJ, Horton NJ, et al. Normal sexual dimorphism of the adult human brain assessed by In vivo Magnetic Resonance Imaging. Cereb Cortex 2001; 11(6): 490-7.

[5] Allen JS, Damasio H, Grabowski TJ, Bruss J, Zhang W. Sexual dimorphism and asymmetries in the gray-white composition of the human cerebrum. NeuroImage 2003; 18: 880-94.

[6] Gur RC, Turetsky BI, Matsui M, et al. Sex differences in brain gray and white matter in healthy young adults: correlations with cognitive performance. J Neurosci 1999; 19(10): 4065-72.

[7] Kaufmann CWM, Putz B, Auer D. Gender differences in brain morphology: Voxel Based Morphometry in 102 healthy young women and men. Proc Intl Mag Reson Med 2001; 9.

[8] Leonard CM, Towler S, Welcome S, et al. Size matters: cerebral volume influences sex differences in neuroanatomy. Cereb Cortex 2008; 18(12): 2920-31.

[9] Marner L, Nyengaard JR, Tang Y, Pakkenberg B. Marked loss of myelinated nerve fibers in the human brain with age. J Comp Neurol 2003; 462(2): 144-52.

[10] Witelson SF, Glezer II, Kigar DL. Women have greater density of neurons in posterior temporal cortex. J Neurosci 1995; 15: 3418- 28.

[11] Pakkenberg B, Gundersen HJ. Neocortical neuron number in humans: Effect of sex and age. J Comp Neurol 1997; 384: 312-20.

[12] Hsu J, Leemans A, Bai C, et al. Gender differences and age-related white matter changes of the human brain: A diffusion tensor imaging study. Neuroimage 2008; 39(2): 566-77 